\title{
Integridad de las empresas e instituciones que impulsan la Responsabilidad Social Corporativa (RSC) en México
}

\author{
Integrity of companies and institutions that promote Corporate Social \\ Responsibility (CSR) in Mexico
}

\author{
Estefania Citlali Monroy Uribe ${ }^{a}$, Alondra Adamary Alvarado López ${ }^{b}$, Perla Lizeth Monroy \\ Sánchez ${ }^{c}$, Martha Azucena Aguilar Lugo ${ }^{d}$, Oropeza Bahena Sandra Areli(e), Adriana Martínez \\ Lecuona ${ }^{f}$.
}

\begin{abstract}
:
Social Responsibility (SR) is a concept that defines a way of acting under ethical principles, aligning itself with sustainable development and appealing to the will of entrepreneurs to innovate, produce without polluting and induce good practices in the company for the well-being of their employees and the environment. The purpose of the work is to identify whether there is integrity on the part of the companies that hold a Socially Responsible Company (ESR) badge in Mexico. There are companies that have been subjected to criticism for their lack of social responsibility. However, the Mexican Centre for Philanthropy (CEMEFI) grants them a distinctive such as ESR. To achieve the objective of this work, the issues that support the problem, Social Responsibility (SR), Corporate Social Responsibility (CSR) and integrity, are analysed, as well as a comparison of the performance of CEMEFI with FORETICA, a Spanish association that issues a certification starting in 2002. After presenting the analysis of some cases of these companies, it is concluded that the integrity of the companies and the institutions that grant distinctions, recognitions or certifications; It is an issue that remains pending to continue questioning and discussing academically.
\end{abstract}

\section{Keywords:}

Social Responsibility (RS), Corporate Social Responsibility (CSR), Integrity.

\section{Resumen:}

La Responsabilidad Social (RS) es un concepto que define una forma de actuar bajo principios éticos, alinearse con el desarrollo sostenible y apelar a la voluntad de los empresarios para innovar, producir sin contaminar e inducir buenas prácticas en la empresa para el bienestar de sus empleados y el entorno. La finalidad del trabajo es identificar si existe integridad por parte de las empresas que ostentan un distintivo de Empresa Socialmente Responsable (ESR) en México. Existen empresas que han sido sujetas a críticas por su falta de responsabilidad social. Sin embargo, el Centro Mexicano para la Filantropía (CEMEFI) les otorga un distintivo como ESR. Para lograr el objetivo de este trabajo se analizan los temas que dan sustento al problema, Responsabilidad Social (RS), Responsabilidad Social Corporativa (RSC) e integridad, así como la comparación de la actuación del CEMEFI con FORETICA, asociación española que emite una certificación a partir del año 2002. Después de presentar el análisis de algunos casos de estas empresas, se concluye que la integridad de las empresas y de las instituciones que otorgan distintivos, reconocimientos o certificaciones; es un tema que queda pendiente de seguir cuestionando y discutir académicamente.

\section{Palabras Clave:}

Responsabilidad Social (RS), Responsabilidad Social Corporativa (RSC), Integridad.

\footnotetext{
Autor de Correspondencia, Universidad Autónoma del Estado de Hidalgo, https://orcid.org/0000-0002-2038-2320, Email: mo326400@uaeh.edu.mx

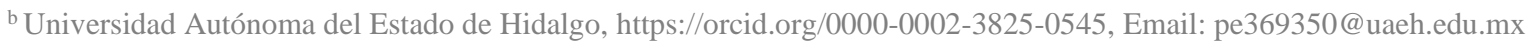

c Universidad Autónoma del Estado de Hidalgo, https://orcid.org/0000-0001-7116-3104, Email: mo368929@uaeh.edu.mx

d Universidad Autónoma del Estado de Hidalgo, https://orcid.org/0000-0003-3627-2449, Email: ag369180@uaeh.edu.mx

e Universidad Autónoma del Estado de Hidalgo, https://orcid.org/0000-0001-9120-0312, Email: or368417@uaeh.edu.mx

f Universidad Autónoma del Estado de Hidalgo, https://orcid.org/0000-0002-5676-5665, Email: alecuona@uaeh.edu.mx
} 


\section{Introducción}

Sabemos que la Responsabilidad Social Corporativa (RSC) actualmente es uno de los temas más importantes que se desarrolla en gran parte de las organizaciones a nivel mundial, por lo que las empresas han tomado la decisión de tener un papel activo para participar con planes integrales de desarrollo, los cuales se llevan a cabo en busca de mejorar las condiciones del macro entorno. Para poder realizar una propuesta integral de RSC las organizaciones deben de contar con principios sólidos y valores. Estos principios y valores crean un compromiso empresarial en busca de la mejora continua, debido a que en las empresas existe una visión a largo plazo con múltiples beneficios para la sociedad y la misma organización.

El término Responsabilidad Social Corporativa (RSC), surge de la relación existente entre la empresa y la sociedad en la que se encuentra. Dentro de este marco social donde existen mayores expectativas y exigencias, la RSC conforma un nuevo modelo de gestión para las organizaciones. Esta misma permite abrir caminos diferentes para trabajar en el ámbito relacional y generar así valor en todos los públicos con los que se comunica.

\section{Responsabilidad Social y Responsabilidad Social Corporativa}

La Comunidad Europea (2000) define la Responsabilidad Social (RS) como el compromiso que se sume por los impactos generados hacia la sociedad.

La variedad de definiciones de responsabilidad social muestra que no existe una percepción única y homogénea de lo que significa. Existen algunos conceptos cercanos a la caridad o donaciones de dinero, tiempo y talento hacia obras sociales. Realmente es un tema que causa mucha inquietud, puesto que las definiciones de RS son diversas, todas estas enfocadas en lo social, económico, ambiental y ético; en donde los actos definen la moralidad de los individuos y la forma en que se hacen responsables.

Desde el ámbito empresarial, la responsabilidad social es el compromiso de una organización ante los impactos que sus decisiones y actividades ocasionan en la sociedad y el medioambiente, a través de un comportamiento transparente y ético (ISO 26000, 2004).

Otros entienden la RS para las empresas, como una estrategia que permita garantizar mejores condiciones laborales a sus trabajadores. En otros casos se describe como impulsos de mejora hacia la comunidad en donde se localizan las empresas, bajo una lógica de adoptar valores éticos. También se le ubica como una estrategia dirigida hacia los stakeholders bajo un enfoque de "ganarganar". Finalmente, otros la perciben como una forma de asumir la dirección estratégica de la empresa de tal forma que constituya un planteamiento integral para el manejo organizacional, como una acción estratégica y de largo plazo articulada a la dinámica interna de la empresa y su entorno (Gómez, 2003).

Por tanto, la RS es el compromiso, obligación y deber que poseen los individuos, miembros de una sociedad 0 empresa, de ayudar voluntariamente para tener una sociedad más justa y de proteger el ambiente.

La RS puede comprender las acciones negativas y positivas, así como la forma de hacerse responsable de ellas. Sin embargo, la RS no es obligatoria pero trae beneficios para todos los involucrados. El cumplimiento de la RS genera ventajas para la sociedad y la organización debido a que aumenta su reputación, fomenta la confianza pública y mejora la salud de los trabajadores y de los individuos que conforman la sociedad.

La responsabilidad social de la empresa es el reflejo de la manera en que las empresas toman en consideración las repercusiones que tienen sus actividades sobre la sociedad, y en la que afirman los principios y valores por los que se rigen, tanto en sus propios métodos y procesos internos como en su relación con los demás actores (Ginebra, 2006)

La Responsabilidad Social Corporativa es una iniciativa de carácter voluntario y que sólo depende de la empresa, y se refiere a actividades que se considera rebasan el mero cumplimiento de la legislación (Cemefi, 2002).

La Responsabilidad Social Corporativa no es independiente a la empresa, actualmente se ha convertido en una nueva forma de gestión y de hacer negocios, en la cual la corporación se ocupa de que sus operaciones sean sustentables en lo económico, lo social y lo ambiental, reconociendo los intereses de los distintos grupos con los que se relaciona y buscando la preservación del medio ambiente y la sustentabilidad de las generaciones futuras. Es una visión de negocios que integra el respeto por las personas, los valores éticos, la comunidad y el medioambiente con la gestión misma de la empresa, independientemente de los productos o servicios que ésta ofrece, del sector al que pertenece, de su tamaño o nacionalidad (Cemefi, 2002).

Dado a esto se comprende que la Responsabilidad Social Corporativa no es algo ajeno a la empresa, requiere del despertar de la conciencia de la forma en que impactará de forma positiva o negativa, directa o indirectamente a los involucrados, sustentándose de valores y plasmándose en la cultura de la empresa. 
La Responsabilidad Social Corporativa ofrece una nueva opción de gestión estratégica, así como también dirige el compromiso que asumen las empresas hacia la sociedad en beneficio del desarrollo sostenible; es decir, pretende equilibrar el crecimiento económico y el bienestar social sostenible en un contexto complejo y sensible (Sarmiento, 2011).

\section{Responsabilidad Social Corporativa en México}

En la historia de la Responsabilidad Social Corporativa (RSC) en México, Tamayo (2015) habla de un primer momento en México a finales del siglo XIX, donde se registró un fuerte auge industrial en Monterrey. En esta ciudad se instaló la Cervecería Cuauhtémoc, la Fundidora de Acero Monterrey y la empresa Vitro. En estas empresas Mexicanas es donde se pone en práctica lo que ahora se maneja como la RSC.

Primordialmente con los empleados, en el año 1918 la Cervecería Cuauhtémoc se constituye como sociedad cooperativa de ahorros e inversiones para los empleados y operarios. De esta manera, la empresa tiene como objetivo proporcionar despensas, así como servicios médicos para su personal y familias. También ofrecía caja de ahorros, descuentos, cursos, becas y por ultimo otorgo créditos para vivienda en casas construidas por la propia empresa. Este tipo de acciones era algo totalmente nuevo tanto en Monterrey como en el resto de México.

Otra fecha conmemorable para la RS fue en la constitución de 1917, en la cual se habla del artículo 123, que señalaba los principales derechos y obligaciones de los trabajadores y empleadores; además de hablar de un salario mínimo. Posteriormente, el gobierno promulga la Ley Federal del Trabajo en 1970. Posteriormente, en 1943, nace el Instituto Mexicano del Seguro Social (IMSS); y finalmente la responsabilidad social se ve reflejada en la creación del Instituto del Fondo Nacional de la Vivienda para los Trabajadores. (INFONAVIT) por parte del gobierno de México en 1972.

Las asociaciones civiles y organismos no gubernamentales en México promueven y apoyan la responsabilidad social corporativa. Pionero es el Centro Mexicano para la Filantropía (Cemefi), que de manera conjunta con la Alianza por la Responsabilidad Social Empresarial en México (AliaRSE), otorgan el distintivo de Empresa Socialmente Responsable (ESR) para acreditar y reconocer a compañías líderes en su compromiso de aportar la responsabilidad social en todos los niveles de su operación.
En México, Lara (2000) indica que del total las empresas que son clasificadas como grandes, el $88 \%$ hacen donativos a causas sociales diversas y el $12 \%$ no. Asimismo, menciona que las razones por las que son otorgadas estas donaciones se deben a la responsabilidad, generosidad, incentivo fiscal o imagen.

Se menciona que las empresas mexicanas se ven presionadas por ayudar de forma social, dado a que el tema es cada vez más abordado en asuntos de ética de los negocios y la Responsabilidad Social es más relevante en estos últimos años, tanto para los consumidores como para las empresas (Cemefi, 2004).

Para el caso del consumidor mexicano, Valdés et al. (1999), afirma que el $74 \%$ de los consumidores estarían dispuestos a comprar preferentemente productos que apoyen causas sociales.

Si realmente la responsabilidad social se llevara a cabo de la forma adecuada, el impacto que se tendría sería positivo para todos los involucrados, ya que deja consigo beneficios como un clima organizacional sano y de confianza entre los interesados.

El transmitir al colaborador la importancia de la diversidad cultural en la organización y respetarla, ayuda a incrementar resultados desde $76 \%$ hasta $94 \%$, de acuerdo a los índices de percepción (Preciado, 2019). Derivado de esto, si invierten tiempo, esfuerzos y recursos para la consolidación de sus prácticas de Responsabilidad Social Empresarial y lo vinculan como una estrategia de negocios, obtendrán los resultados deseados.

El involucrar la Responsabilidad Social para las empresas no es nada sencillo. Se debe de iniciar con un cambio de cultura, tanto de los directivos como de los empleados, para después tener cambios radicales que van desde la filosofía del negocio hasta las más pequeñas actividades que se realizan en todas las áreas funcionales. Esto es una gran tarea que efectivamente, no todas las empresas quieren realizarlo debido a que exige hacer lo correcto y no solo decirlo.

\section{Centro Mexicano para la Filantropía (Cemefi)}

El Centro Mexicano para la Filantropía (Cemefi) es una asociación civil fundada en diciembre de 1988. El Cemefi es una asociación no lucrativa, cuenta con el permiso del Gobierno Federal para recibir donativos deducibles de impuestos y no tiene filiación de partidos políticos, raza o 
religión. Esta institución busca la promoción y articulación de la participación filantrópica, comprometida y socialmente responsable de los ciudadanos, sus organizaciones sociales y empresas para alcanzar una sociedad más equitativa, solidaria y próspera (Cemefi, 2002).

Para México y sus empresas, esta organización se piensa que es la más importante para realizar el análisis del perfil de empresas que se presumen como socialmente responsables.

Para el análisis de la RSC, en México se ha considerado comúnmente con el término Responsabilidad Social Empresarial (RSE), según como lo refiere el Centro Mexicano para la Filantropía (Cemefi, 2002).

Cemefi entiende por Responsabilidad Social "la conciencia del compromiso y la acción de mejora continua, medida y consistente, que hace posible a la empresa ser más competitiva, cumpliendo con las expectativas de todos sus participantes (Calderón, 2002).

Para el Cemefi (2002), la aplicación de la RS en el ámbito empresarial implica los siguientes criterios:

- La RSE es un acto voluntario.

- La RSE debe ser integrada mediante el uso de técnicas sustentables en la producción y el uso de los recursos naturales, como mecanismo de actuación socialmente responsable dentro de la empresa.

- La RSE debe enfocarse en lo económico, social y ambiental y va dirigida a todos los Grupos de Interés (Stakeholders).

El Cemefi tiene el compromiso consciente y congruente de cumplir íntegramente con verificar la finalidad de la Responsabilidad Social de la empresa, tanto en lo interno y lo externo, la cual considere las expectativas de todos sus participantes en lo económico, social o humano y ambiental, demostrando el respeto por los valores éticos, la gente, las comunidades, el medio ambiente y para la construcción del bien común (Gómez, 2003).

Por su parte, el Cemefi define el distintivo Empresa Socialmente Responsable (ESR) como un proceso de confianza, honestidad y transparencia empresarial; cuyos indicadores son afines a lineamientos nacionales $e$ internacionales, lo cual garantiza su validación frente a organismos internacionales, como son: Global Compact Principales (UN), Business in the Community, Caux Round Table Principles, Código de Mejores Prácticas Corporativas (CCE), Convenio de lucha contra la Corrupción (OCDE), Great Place to Work, Declaración
Universal de los Derechos Humanos, Declaración de Principios Fundamentales y Derechos del Trabajo (OIT).

Desde que se implantó este distintivo en el año 2001, las empresas mexicanas que lograron obtenerlo se ha incrementado. De aquí en adelante se puede observar que el Cemefi tiene una importante representación a lo que se refiere la responsabilidad social en México.

Los criterios para la evaluación que realiza el Cemefi han permitido que las empresas en México; y otros países de Latinoamérica, logren ostentar dicho distintivo el cual se ha convertido en un gran aliento para que la sociedad sepa que algunos negocios están preocupados por cumplir con su Responsabilidad Social (Celis, Torres \& Castro, 2012).

Es así como las empresas que obtienen el reconocimiento de Empresa Socialmente Responsable (ESR) se vuelven mucho más competitivas ante la sociedad, dejándonos la esperanza de que éstas se manejan adecuadamente, lo cual genera una imagen corporativa buena a la vista de sus consumidores.

\section{Lo que se espera de una empresa con responsabilidad social}

Chambers, (1999) define la integridad como el apego estricto a los valores y principios morales. En el ámbito organizacional, la integridad se refiere a la capacidad de las empresas de desarrollar e implementar un marco de gestión de la integridad y que sus empleados actúen de acuerdo con los valores de la organización (Matten, Pohl y Tolhurst, 2007). Es así como las empresas que son Socialmente Responsables deben enfocar sus actos en acciones íntegras y que sean realmente congruentes de lo que hacen, con lo que dicen.

Cada vez es más grande la cantidad de ejecutivos, propietarios de empresas y directores que buscan incorporar la responsabilidad social dentro de su estrategia empresarial, la cual se conoce como estrategia de responsabilidad social. De esta forma, ellos comprenden que sus actos y acciones tienen cierto impacto en la sociedad, ya sea de forma positiva o negativa; y que es preferible impactar de manera adecuada para construir una mejor sociedad de forma sustentable (Rochlin, 2005).

No se busca maximizar simplemente las utilidades, si es a causa de la violación de los derechos laborales, de la degradación de nuestro planeta o el uso de acciones éticas cuestionables; se busca ser más cuidadoso con lo que se hace dentro y fuera de la empresa, tratando de 
incorporar a cada uno de los involucrados, e incorporar en ellos un patrón repetitivo de buenas prácticas.

Realmente la integridad de una empresa es un tema muy extenso que sigue en discusión por muchos autores, es algo que comenzó a volverse tendencia. Hace algunos años Rochlin (2005) indicaba que había confusión sobre el alcance que tiene la responsabilidad social, puesto que hay personas o ejecutivos que la confunden con actos de buena voluntad. Estos actos son como la creación de escuelas en áreas donde opera la empresa, la donación de dinero a fundaciones culturales o artísticas etc., solo para contar una imagen o reputación adecuada. Sin embargo, todos estos buenos actos tienen su lado opuesto porque contaminan y explotan a personas que laboran en las empresas.

Lo anterior hace que realmente nos cuestionemos si son empresas con responsabilidad social, o simplemente buscan una buena imagen ante la sociedad.

Se debe considerar que las empresas que asumen su responsabilidad social incluyen en ellas cuatro ámbitos que son los siguientes (Gómez, 2003):

1. Contribuir a la calidad de vida dentro de la empresa. De acuerdo con Barroso (2007), estas organizaciones deben generar empleos, sueldos y salarios que realmente cumplan los requisitos, conforme a la ley federal del trabajo, ayudando en cierta parte al desarrollo de sus trabajadores y ofreciéndoles calidad de vida; al mismo tiempo, sus empleados con sus ingresos contribuirán al desarrollo de las comunidades donde cada uno de ellos radica. Las empresas que cumplen con este ámbito tienen un clima organizacional y calidad de vida laboral, esto con ayuda de constantes capacitaciones, una supervisión concreta, comunicación adecuada y un trato digno hacia su persona. Todo esto si se genera de una manera correcta tendrá un impacto positivo, dado a que se logrará un aumento de la autoestima, mejor manejo de las finanzas personales, trabajo en equipo, así como la prevención de adicciones y accidentes de trabajo.

2. Cuidado y preservación del medio ambiente. Es realmente necesario que las empresas se preocupen por cuidar el entorno que les rodea y fomenten, entre todos sus grupos de interés, el interés de preservarlo junto con sus familias. No es suficiente solo el cumplimiento de las normas ambientales, si no que al igual se deben generar valores que perduren de manera permanente y voluntaria; y de ser posible, involucrar a personas especializadas en los temas del medio ambiente.
3. Desempeñarse con un código de ética. Tener un trato ético ayudará a las empresas a mantener relaciones de calidad tanto con sus empleados, como proveedores y clientes. Esto tendrá como resultado que sus grupos de interés (stakeholders) buscarán tener con ellos muchos más una relación económica. En consecuencia, se incrementarán los niveles de competitividad en la empresa y tendrán como efecto una mejor calidad en sus relaciones. Esto es una relación ganar-ganar, o de ganancia óptima (Barroso, 2008).

4. Vincularse con la comunidad a partir de la misión del negocio, pero también de los bienes y servicios producidos. Se espera que las empresas que son socialmente responsables fomenten el desarrollo de la comunidad y así mismo ayuden en causas sociales y de bienestar público, más allá de la filantropía.

Para que una empresa realmente sea responsable socialmente debe de cumplir con estos cuatro ámbitos a la vez, si solo practica alguno de ellos, quiere decir que solo están realizando acciones de compromiso empresarial con la sociedad, pero no quiere decir que son socialmente responsables en el sentido amplio del concepto.

Las empresas socialmente responsables buscan dar a conocer, asumir y adoptar los indicadores que señalan el grado de acción de la responsabilidad social que tienen. Estas empresas deben de respetar los derechos humanos y laborales, tener cuidado y conservar el medio ambiente, tomando en cuenta a las generaciones futuras. Al momento de hacer un bien común, estas empresas generaran riqueza de una manera adecuada.

Las empresas que enfocan su camino hacia la responsabilidad social, deben aprender a incorporar estrategias en donde aborden acciones de negocios responsables; al enfocarse en la creación de estas obtendrán una ventaja competitiva y contribuirán a su éxito a largo plazo (Zadek, 2005).

Para alinear la responsabilidad social a la estrategia empresarial, se debe tener un compromiso con los grupos de interés. Este compromiso no debe tener como objetivo prevenir crisis o suavizar críticas con los grupos de interés; si no de contribuir con la sociedad y promover el aprendizaje y la innovación dentro de la empresa que impulsará el desempeño del corporativo. 
En la Responsabilidad Social Corporativa interviene de manera significativa el liderazgo, donde la intención es sumar a los directivos, jefes o responsables de las diferentes áreas en la organización a contar con habilidades y capacidad para conducir a su empresa a enfrentar eficazmente los problemas sociales y ambientales desafiantes.

A pesar de que esto es algo que una empresa socialmente responsable debe llevar a cabo, muchas de ellas están lejos de asumir su compromiso con la responsabilidad social, puesto que buscan simplemente hacerse de publicidad para elevar sus ganancias y vender más productos, o utilizan este concepto simplemente para poder justificar un fin bueno con un principio negativo para el hombre.

Es así como nacen algunos cuestionamientos sobre el objetivo y desempeño del Cemefi con relación a: ¿Es una asociación de carácter social y sin fines de lucro realmente? ¿El distintivo de Empresa Socialmente Responsable (ESR) lo otorga a las empresas que son verdaderamente responsables? ¿Garantiza el cumplimiento de la responsabilidad social de las empresas que han mantenido el distintivo en los siguientes años al que éste fue otorgado?

La respuesta a estas preguntas se hace necesario debido a que el Cemefi se ha visto envuelto en noticias un tanto polémicas poniendo en duda la confiabilidad del distintivo de Empresa Socialmente Responsable. Ejemplo de esto fue publicado en el primer medio de comunicación sobre Sustentabilidad y Responsabilidad Social Empresarial de habla hispana, fundado en 2002 llamado "Comunicarse".

Este medio de comunicación cuestiona si el Cemefi está haciendo realmente lo correcto en relación a la forma en que las empresas y corporativos se someten a "autoevaluación" para obtener el distintivo de Empresa Socialmente Responsable (ESR). En este sentido, Díaz afirma que debiera mediar una auditoría que no nada más atienda lo que les está diciendo la empresa en su autoevaluación, porque ahí todos son buenos. Se trata de conocer el verdadero actuar de la compañía, qué es lo que aporta y cómo se maneja (Díaz s/f, Citado por comunicarSe, 2012).

Comentarios como este y otros fueron mencionados en diversos medios periodísticos, dado a que observan que las empresas con este distintivo son las mismas que tienen un alto poder adquisitivo; y que fácilmente podrían realizar actos poco éticos con el fin de obtener una imagen corporativa que atraiga a todos sus consumidores. Sin embargo, existe contradicción ocasionada por ciertas acciones que realizan estas empresas en cuanto al cuidado del medio ambiente, la responsabilidad con sus trabajadores y la sociedad.
Esto hace recordar que las empresas que realmente son socialmente responsables deben de cumplir con ciertos actos morales, que dejen en claro que fueron realizados de una manera transparente; no contradiciendo lo que dicen con lo que hacen. Es fundamental crear conciencia de esto para formar criterios enfocados en la esencia que representa la Responsabilidad Social Corporativa (RSC).

\section{La imagen de las empresas que obtuvieron el distintivo de Empresa Socialmente Responsable (ESR) en México}

Cemefi otorga el distintivo de Empresa Socialmente Responsable (ESR) a empresas que considera, según sus lineamientos, que lo merecen. Estas empresas obtienen con ello una ventaja competitiva en cuanto a que sus clientes la ven más atractivas por llevar a cabo la Responsabilidad Social. Sin embargo, lo que realmente se considera importante es dejar en evidencia si las empresas que obtuvieron el distintivo de Empresa Socialmente Responsable (ESR) practican lo que realmente significa la responsabilidad social.

Para realizar el análisis se presentan algunos casos de empresas que han obtenido el reconocimiento como empresas socialmente responsables (ESR) en México, con relación a las actividades que llevan a cabo y el impacto que generan en la sociedad a través de los programas.

Los datos que publico Cemef (2020), en relación a las empresas que obtuvieron el distintivo en el año 2020 son:

- $\quad$ BBVA

- Deloitte

- Hewlett Packard Enterprise México

- HP Inc. México

- Walmart de México y Centroamérica.

- Coca - Cola de México

- Grupo Bimbo, S.A.B. de C.V.

- Holcim México

- Shell

- Zimat Consultores

Entre las empresas que se enlistan, Grupo Bimbo declara que "Desde nuestra fundación asumimos con firme convicción, valores y ejes que guían los proyectos y metas de Grupo Bimbo para atender con responsabilidad a nuestros diferentes grupos de interés y a la sociedad en general. Todo esto lo llevamos a cabo a través de nuestros 4 programas de responsabilidad social: comprometidos 
con tu salud, comprometidos con el medio ambiente, comprometidos con nuestros colaboradores y comprometidos con nuestra sociedad. [...] La satisfacción de apoyar con nuestra pequeña semilla y de participar en esta suma de trabajo responsable, es una razón más para que en Grupo Bimbo continuemos con nuestro compromiso en beneficio de nuestro entorno" (Bimbo, 2017).

Desafortunadamente las y los consumidores no han visto reflejada la responsabilidad, que la empresa asegura tener en acciones reales que protejan el medio ambiente y garanticen alimentos de mejor calidad, ya que Grupo Bimbo ha ignorado la exigencia de más de 60 mil personas por un compromiso a transitar a un modelo de agricultura ecológica a lo largo de su cadena de valor (Lasso, 2016).

Por si esto no fuese suficiente, muestra de su incongruencia y razón para exigirle acciones reales y un compromiso con el medio ambiente y la gente, se presentan otros argumentos.

Grupo Bimbo se esconde tras la legislación mexicana sobre el uso de agro tóxicos que no ha sido actualizada en más de una década, pese a que en público su director ha señalado que la empresa tiene la responsabilidad de garantizar una producción limpia y de calidad (Lasso, 2016).

Asimismo, Bimbo estableció la meta de reducir sus emisiones de bióxido de carbono $\left(\mathrm{CO}_{2}\right)$ de sus plantas, pero no de la agricultura industrial de la que se abastece que contribuye con el $12 \%$ de las emisiones de gases de efecto invernadero en el país (Lasso, 2016).

Otro caso es el de Coca-Cola de México, empresa que a través de su plataforma de sustentabilidad muestra lo que para ellos significa "Viviendo Positivamente", lo que señalan que marca una diferencia tangible en el mundo y asegura un crecimiento sostenible tanto para las comunidades a las que sirve como para su negocio con la implementación de estrategias innovadoras en cada uno de sus campos de acción (FEMSA, 2019).

Su labor en 2010, se enfocó en promover acciones que permitan elevar los estándares de ejercicio físico y fomentar la convivencia familiar, desarrollando más de 2,300 eventos deportivos a los que asistieron cerca de 25 millones de personas. Asimismo, benefició a 4.3 millones de mexicanos con sus proyectos comunitarios. Coca-Cola es uno de los principales empleadores del país al generar más de 93,000 empleos formales directos, además ha sido reconocida como una de las mejores compañías para trabajar (Catarelli, 2021).
Sin embargo, un diario británico investigó los efectos sobre el océano de las botellas de plástico de Coca Cola. Según el corresponsal para el medio ambiente del diario, Tom Bawden, el daño que causa la compañía a los océanos es inaceptable (Cooper, 2017)

Coca Cola, que posee 500 diferentes marcas, produce cada año más de 100 mil millones de botellas de plástico. Aunque Coca Cola no declara una cifra sobre su producción de botellas de plástico, la investigación de Bawden revela que la compañía vende cada segundo 3,500 botellas de plástico (TRT, 2017).

Otro ejemplo claro de esto es Wal-Mart, empresa que obtuvo su distintivo de ESR, por el hecho de contribuir significativamente al crecimiento sostenible de su compañía, cuidado del medio ambiente, el factor social y de su gobierno corporativo. Wal-Mart se dice ser la empresa con autoservicio líder en consumo de energías limpias, promover políticas y prácticas a favor de la diversidad e inclusión, tener estándares éticos y legales más altos sobre su estrategia de negocio a largo plazo.

Esto la ha hecho llevar el distintivo por 19 años consecutivos, pero sus acciones son algo contradictorias, puesto que en el año 2018 hubo huelgas en algunas de las tiendas de Wal-Mart en Estados Unidos. Los trabajadores de las sucursales explicaron que estos paros realizados se debían a los malos tratos, los bajos sueldos, las humillaciones y la discriminación de las que eran objeto (Martin, 2018).

Con estos ejemplos queda claro que la filantropía sigue siendo influencia que busca el beneficio político y económico, proporcionando donaciones y financiamientos en programas y obras que implementan, sin consultar a las personas de la sociedad que se ve afectada por estas condiciones. La Responsabilidad Social Empresarial entendida como compromiso y/u obligación de reconocer y de remediar las consecuencias que tienen las acciones y decisiones hacia la sociedad y el medio ambiente, está muy alejada de la realidad mexicana.

\section{Responsabilidad social empresarial en España}

En el ámbito español, en el marco de la Unión Europea (UE), la Asociación Española de Contabilidad y Administración de Empresas (AECA, 2004, p.21) define la $\mathrm{RSC}$ como el "compromiso voluntario de las empresas con el desarrollo de la sociedad y la preservación del medio ambiente, desde su composición social y un comportamiento responsable hacia las personas y grupos sociales con quienes se interactúa, centrando su atención 
en la satisfacción de las necesidades de los grupos de interés".

Los elementos conceptuales que AECA identifica en la responsabilidad social son:

- Responsabilidad: capacidad para reconocer, aceptar y, en su caso, plantear respuestas a las consecuencias de un comportamiento realizado consciente y libremente

- Social: cualidad de la persona como elemento integrante de un sistema social o colectivo de individuos.

- Corporación: entidad de interés público, comúnmente asociada a un proyecto económico.

- Compromiso voluntario: obligación contraída por voluntad propia.

- Organización: conjunto de personas estable, institucionalizado y estructurado adecuadamente, dotado de medios para alcanzar unos fines.

- Sociedad: agrupación de personas, que constituyen unidad distinta de cada uno de sus individuos con el fin de cumplir, mediante mutua cooperación, todos o alguno de los fines de la vida.

- Medio ambiente: entorno físico natural, incluidos el aire, agua, tierra, flora, fauna y recursos no renovables.

- Persona: individuo, humano.

- Grupos de interés: partes interesadas, o stakeholders, afectados de una u otra manera por la existencia o acción de las organizaciones y con algún interés sobre ellas.

A diferencia de lo que ocurre en México con CEMEFI, AECA no propone un patrón propio, sino que apoya explícitamente la emisión de la información sobre responsabilidad social que emiten las empresas.

En este sentido, el gobierno de España, para dar un impulso al desarrollo de la RS, creó el Foro de Expertos de RSE, cuyo objetivo durante este año es que estudie, debata y proponga medidas para fomentar las prácticas de ética social y medioambiental, dentro del marco del desarrollo sostenible definido por las instituciones de la Unión Europea. Como resultado del trabajo de este Foro, compuesto por representantes de distintos ministerios y expertos provenientes de organizaciones de la sociedad civil y de la Universidad, es su concepción de la responsabilidad social de la empresa aquella que tiene por objetivo "La sostenibilidad basándose en un proceso estratégico e integrador en el que se vean identificando los diferentes agentes de la sociedad afectados por las actividades de la empresa" [...] "para su desarrollo deben establecerse los cauces necesarios para llegar a identificar fielmente a los diferentes grupos de interés y sus necesidades, desde una perspectiva global y se deben introducir criterios de responsabilidad en la gestión que afecten a toda la organización y a toda su cadena de valor" (Crook, 2005).

Por otra parte, en el año 2000 se creó en España una asociación sin ánimo de lucro denominada FORETICA para que fuera un foro multidisciplinar de la responsabilidad social. Uno de sus primeros frutos fue la elaboración de la Noma de Empresa SGE 21, en la que participaron más de 100 expertos representando a todos los grupos de interés.

Esta norma, lanzada en 2002, recoge el primer sistema de gestión de la responsabilidad social europeo que permite, de manera voluntaria, alcanzar una certificación (Urtiaga y Granda, 2001). La SGE 21 es considerada un sistema objetivo de implantación, evaluación y verificación de la gestión ética en las organizaciones.

\section{Conclusiones}

Como finalidad de la Responsabilidad Social Corporativa, podemos deducir que se basa en analizar que impacto tiene el Cemefi en México, si en realidad practica fines de lucro o no puesto que es la organización más importante en México relacionado con la RSE, el Cemefi promueve los derechos de los consumidores tomando en cuenta su bienestar y conformidad de lo que las empresas quieren ofrecer al público.

El Cemefi presume de llevar a cabo la filantropía pero más bien parece actuar por interés de por medio, sabemos que las empresas participan por el distintivo RSE porque su adaptación de esa práctica les da ganancias y mejor imagen en el mercado, los hace ser eficientes y con calidad puesto que agrega valor y da rentabilidad.

Aunque reconociendo que el Cemefi muchas ocasiones le dan el distintivo a empresas que realmente no saben practicar lo que realmente significa la responsabilidad social.

Por otra parte, después de revisar el concepto de responsabilidad social, tanto en México como en España para averiguar la situación actual de su implantación en ambos países podemos establecer las siguientes comparaciones:

1) Aún no se ha logrado una definición de responsabilidad social comúnmente aceptada, ni 
existe un consenso en cuanto a sus objetivos y elementos. En España y México se entiende que la responsabilidad social se relaciona con la adhesión a las diferentes iniciativas de forma voluntaria en los ámbitos medioambiental, social y ético, corroborando la importancia, cada vez mayor, que está adquiriendo la responsabilidad social en el mundo empresarial.

2) Tanto en México como en España, existen distintivos otorgados por instituciones de carácter privado que certifican la implantación de la RS. En México, la iniciativa de Cemefi promueve el distintivo de empresa socialmente responsable (ESR), que han logrado un total de 84 empresas en 2005.

3) Si atendemos a las adhesiones a una iniciativa internacional, como es el Pacto Mundial de la ONU, y según los datos suministrados por el propio Global Compact (2018), México con 7 empresas adheridas presenta un número inferior al de España, que agrupa a 231 empresas adheridas.

4) Además, si complementamos la información obtenida directamente del Global Compact (2018), con otra referida a empresas en proceso de adhesión suministrada por instituciones como CEMEFI, que señala que son 84 las empresas adheridas 0 en proceso de adhesión al Pacto Mundial, como Comité Nacional de Productividad e Innovación Tecnológica (COMPITE) (2021), que reporta que son cerca de 250 empresas las que se hallan en este proceso.

5) El Pacto Mundial-España (2015), reporta que son 46 empresas más las adheridas de las que recoge la página del Global Compact, lo que alcanzó un total de 277 empresas.

Como se puede observar, la Responsabilidad Social en ambos países, así como su reciente evolución, se concluye que existe una evolución rápida y favorable a su implantación; en consecuencia existe un compromiso ético cada vez mayor de las empresas con su entorno social, medioambiental y con los grupos de allegados, a pesar de la visión crítica que algunos autores intentan dar de la responsabilidad social. No obstante la integridad de las empresas y de las instituciones que otorgan distintivos, reconocimientos o certificaciones; es un tema que queda pendiente de seguir cuestionando y discutir en el ámbito académico.

\section{Referencias}

[1] Asociación española de contabilidad y administración de Empresas (2004), marco conceptual de la responsabilidad social corporativa, Documento AECA, Madrid.

[2] Barroso, f. (2007, septiembre-diciembre), Responsabilidad social empresarial: concepto y sugerencias para su aplicación en empresas constructoras, Ingeniería, Revista Académica de la Facultad de Ingeniería, Universidad autónoma de Yucatán, 11, 3, 65-72.

[3] Barroso, f. (2008, febrero), Ganancia máxima o ganancia óptima, Desarrollo empresarial. Año xi, 110, pp. 35-38.

[4] Caldera Sánchez-capitán, j. (2005), Notas de saludo al foro de expertos de RSE, en http://www.mtas.es/empleo/economiasoc/noticiasdoc/noticiasportada/saludoforoexpertos.htm

[5] Calderón, G. (2002). El concepto de Responsabilidad Social.Recuperado el 20 de abril del 2021 de: https://www.cemefi.org/esr/images/stories/pdf/esr/concepto_esr.pdf

[6] Castromán Diz, J. L. \& N. Porto Serantes (2005), Responsabilidad social y control interno, Revista universo cantábile, 1, 2, 86-101.

[7] Catarrelli A. (2021). Coca-Cola de México Recibe el distintivo de ESR, The Food Tech, Recuperado el 20 de abril del 2021 de: https://thefoodtech.com/historico/coca-cola-de-mexico-recibe-eldistintivo-esr/

[8] Celis, L., Torres, A., \& Castro, M. (2012). Analizando las normas en que se basan las acciones de responsabilidad social empresarial (RSE) en México. Contabilidad y auditoría, 28.

[9] Cemefi (2002); Decálogo de la Empresa Socialmente Responsable, Responsabilidad Social, Recuperado el 20 de abril del 2021 de: http://www.cemefi.org/index.cfm?page=CEM_RSE_39\#c

[10] Cemefi (centro mexicano para la filantropía) (2004), en, 11 de febrero de 2021. Compite. Recuperado. 23 de abril del 2021, de Org.mx website: https://www.compite.org.mx

[11] Cemefi. (2020). empresas que obtuvieron el distintivo en el año 2020, Centro Mexicano para la Filantropía,Recuperado el 20 de abril del 2021 de: https://www.cemefi.org/

[12] Chambers 21st Century Dictionary (1999). integrity, Edinburgh, Chambers.Diccionario Chambers Siglo XXI,

[13] ComunicarSE. (2012). Escándalos corporativos siembran dudas sobre el Distintivo ESR de CEMEF, ComunicarSe, Recuperado el 20 de abril del 2021 de: https://www.comunicarseweb.com/biblioteca/escandaloscorporativos-siembran-dudas-sobre-el-distintivo-esr-de-cemefi

[14] Comunidad Europea, (2000), El futuro está en nuestras manos, DG Medio Ambiente, Recuperado el 20 de abril del 2021 de: http://europa.eu.int/comm.environnement/newprogr/index.htm

[15] CROOK C. (2005), The good company, Economist, 22 de enero de 2005, 374, (8.410), 3-5.

[16] FEMSA (2019), Informe Anual, FEMSA, Recuperado el 20 de abril del 2021 de: https://www.informeanual.femsa.com/ x0007_x0012_x0010_x0012

[17] Fong. C, Parra. A, Soriano. L. \& Teodoro. E. (2020). The current situation of social corporate responsibility in Mexico. Universidad de Guadalajara, centro universitario de ciencias económico administrativas (CUCEA). Jalisco, México. Consultado: 11/02/21 disponible en: Revista Académica y Negocios 6(1).

[18] Ginebra. (2006). Iniciativa InFocus sobre responsabilidad social de la empresa,Consejo de Administración, Recuperado el 20 de abril del 2021 de: https://www.ilo.org/public/libdoc/ilo/GB/295/GB.295_MNE_2_1_spa n.pdf 
[19] Gómez, M. \& Calvo, A. (2004). Globalización, desarrollo sostenible y empresa: Virando hacia la responsabilidad social. Revista Lúmina, 5, 209-236.

[20] González Santos. (2007), Responsabilidad social empresarial: un análisis comparativo de la iniciativa del Cemefi y las iniciativas internacionales. Tecnológico de monterrey. Estado de México, México. Consultado el 05/02/2021.disponible en: https://repositorio.tec.mx/handle/11285/569090

[21] Grupo Bimbo. (2017). Grupo Bimbo reafirma su compromiso con el medio ambiente, Grupo Bimbo Recuperado el 20 de abril del 2021 de: https:/grupobimbo.com/es/sala-de-prensa/comunicados/grupo-bimboreafirma-su-compromiso-con-el-medio-ambiente

[22] Irenix, P. \& Lorenzo, A. (2015, 18 de marzo). Pacto Mundial Responsabilidad Social Empresarial - RSE - Objetivos de Desarrollo Sostenible - ODS - Derechos Humanos y Empresa. Obtenido el 23 de abril de 2021 del sitio web Pactomundial.org: https://www.pactomundial.org/

[23] ISO 26000, (2004), Guía de Responsabilidad Social, Online Browsing Platform (OBP), Recuperado el 20 de abril del 2021 de: https://www.iso.org/obp/ui\#iso:std:iso:26000:ed-1:v1:es

Lara, M. (2000), Filantropía empresarial: convicción y estrategia, México, pax.

Matten, Pohl \& Tolhurst. (2007). Integridad y Étic, Marco Conceptual Recuperado el 20 de abril del 2021 de: https://www.unodc.org/documents/e4j/IntegrityEthics/MODULE_1__Introduction_and_Conceptual_Framework_-_Spanish.pdf

Martin, M. (2018, abril), aspectos negativos de Wal-Mart extraído de: https://es.scribd.com/document/376375293/aspectos-negativos-dewalmart

[27] Mazzotti Pabello. G.M., Aguilar Pizarro. V. \& Vargas Rubín. H. J. (2016). Corporate social responsibility (CSR) in Mexico: the influence of cultural values, leadership, institutional context and national "style" in normalization and implementation). Universidad Veracruzana. Veracruz, México. Consultado 11/02/21

[28] Paulo Cesar Preciado, (2019), El impacto de la Responsabilidad Social Empresarial en los colaboradores, Responsabilidad Social y Sustentabilidad, Recuperado el 20 de abril del 2021 de: https://ganarganar.mx/2019/10/02/el-impacto-de-la-responsabilidad-socialempresarial-en-los-colaboradores-2/

[29] Rivas, S. (2021). Ayuda en tramites online en México - Tramites INFONAVIT, RFC, CURP, SFE, SEMS, IMSS. (2021, March 25).

[30] Rochlin, S. (2005, agosto), Llevar la responsabilidad corporativa al ADN de su empresa", Harvard Review, 83(8), 31-38.

[31] Salazar, Sabrina (2004), "La responsabilidad social corporativa en México como una herramienta estratégica para la competitividad: un enfoque econométrico y prospectivo", Monterey Mexico, Economía, Sociedad y Territorio ISSN: 1405-8421

[32] Sandra Lasso. (2016). La incongruencia del Grupo Bimbo, Sin embargo.mx, Recuperado el 20 de abril del 2021 de: https://www.sinembargo.mx/20-06-2016/3056313

[33] Sarmiento, S. (2011). La responsabilidad social empresarial: gestion estrategica para la supervivencia de las empresas. Dialnet, 9(2), 6-15. Recuperado el 11 de febrero de 2021, de https://dialnet.unirioja.es/descarga/articulo/3965840

[34] Tamayo Neira A. (2015). La responsabilidad social en México, disponible en: ecologistasocial.com.mx

[35] The UN Global Compact and Accenture Strategy. (2018). Transforming Partnerships for the SDGs. Special Edition: The UN Global Compact-Accenture Strategy CEO Study. Disponible en https://www.accenture.com/_acnmedia/PDF-74/AccentureTransforming-Partne

TRT. (2017). El daño que causa Coca Cola al medio ambiente es "chocante". TRT español. Recuperado el 20 de abril del 2021 de:
Recuperado el 20 de abril del 2021 de: https://www.trt.net.tr/espanol/vida-y-salud/2017/04/10/hallan-restosde-1-646-yazidies-en-mosul-709280

Zadek, S. (2005, agosto), El camino hacia la responsabilidad corporativa, Harvard Business Review, 83(8), 60-69. 